06,11

\title{
Протонпроводящие композиционные материалы на основе соединения $\mathrm{Cs}_{6} \mathrm{H}\left(\mathrm{HSO}_{4}\right)_{3}\left(\mathrm{H}_{2} \mathrm{PO}_{4}\right)_{4}$
}

\author{
() В.А. Коморников, В.В. Гребенев, И.С. Тимаков, О.Б. Зайнуллин
}

${ }^{1}$ Институт кристаллографии им. А.В. Шубникова РАН,

Федеральный научно-исследовательский центр „Кристаллография и фотоника“ РАН, Москва, Россия

E-mail: v.a.kom@mail.ru

Поступила в Редакцию 16 июля 2019 г.

В окончательной редакции 16 июля 2019 г.

Принята к публикации 25 июля 2019 г.

Получены композиционные протонпроводящие материалы $x \mathrm{Cs}_{6} \mathrm{H}\left(\mathrm{HSO}_{4}\right)_{3}\left(\mathrm{H}_{2} \mathrm{PO}_{4}\right)_{4}+(1-x) \mathrm{AlPO}_{4} \quad$ в интервале составов $(x=0.9 \div 0.7)$. Методами рентгенофазового анализа, импедансной спектроскопии, электронной сканирующей микроскопии изучены их физико-химические и транспортные свойства.

Ключевые слова: Кристаллы-суперпротоники, фазовые переходы, композитные материалы.

DOI: $10.21883 /$ FTT.2019.12.48557.16ks

\section{1. Введение}

Перспективными для использования в качестве материалов для водородно- воздушных топливных элементов являются соединения семейства с общей формулой $\mathrm{M}_{\mathrm{m}} \mathrm{H}_{\mathrm{n}}\left(\mathrm{XO}_{4}\right)_{(\mathrm{m}+\mathrm{n}) / 2} \cdot y \mathrm{H}_{2} \mathrm{O}\left(\mathrm{M}=\mathrm{K}, \mathrm{Rb}, \mathrm{Cs}, \mathrm{NH}_{4}\right.$, $\left.\mathrm{XO}_{4}=\mathrm{SO}_{4}, \mathrm{SeO}_{4}, \mathrm{HPO}_{4}\right)$. Одним из уникальных свойств кристаллов этого семейства, названного суперпротониками, является аномально высокая протонная проводимость при относительно невысокой температуре.

О перспективности использования материалов на основе кристаллов-суперпротоников топливных элементов на водороде упоминалось в ведущих научных журналах „Nature“ [1], „Physics Today" [2].

В то же время ограничивает применение соединений этого семейства как раз необходимость получения функциональных материалов в виде тонких мембран с низкой газопроницаемостью. Очевидным решением данной проблемы является использование композитных материалов.

Таким образом, данная работа является логическим продолжением ранее полученных результатов и посвящена получению функциональных протонпроводящих материалов, а именно определению условий синтеза композиционных материалов переменного состава $x \mathrm{Cs}_{6} \mathrm{H}\left(\mathrm{HSO}_{4}\right)_{3}\left(\mathrm{H}_{2} \mathrm{PO}_{4}\right)_{4}+(1-x) \mathrm{AlPO}_{4}$ (полиалюмофосфат) в интервале составов $x=0.9 \div 0.7$; анализу физико-химических свойств этих материалов.

\section{2. Экспериментальная часть}

В работе для получения монокристаллов $\mathrm{Cs}_{6} \mathrm{H}\left(\mathrm{HSO}_{4}\right)_{3}\left(\mathrm{H}_{2} \mathrm{PO}_{4}\right)_{4}$ использовали гидросульфат цезия (CsHSO4) марки „ч“", ТУ 6-09-04-198-83 и дигидроортофосфат цезия $\left(\mathrm{CsH}_{2} \mathrm{PO}_{4}\right)$ марки „хч“,
ТУ 6-09-04-201-82 без дополнительной очистки и кислота фосфорная $85 \% \mathrm{H}_{3} \mathrm{PO}_{4}$ „ЧДА“, ГОСТ 6552-80. Для получения композиционных материалов использовали: алюмоаммонийные квасцы „осч 7-4“ $\mathrm{Al}\left(\mathrm{NH}_{4}\right)\left(\mathrm{SO}_{4}\right)_{2} \cdot 12 \mathrm{H}_{2} \mathrm{O}, \quad$ TУ 6-09-3717-76; раствор аммиака водного особой чистоты „осч. 25-5“ $\left(\mathrm{NH}_{3} \cdot \mathrm{H}_{2} \mathrm{O}\right)$, ГОСТ $24147-80$ и ранее упомянутая фосфорная кислота.

Рентгенофазовый анализ (РФА) размолотых в порошок образцов материалов выполнен при комнатной температуре на настольном рентгеновском дифрактометре Rigaku Miniflex 600 (Япония) (рентгеновская трубка с медным анодом, непрерывный режим съемки $2 \mathrm{grad} / \mathrm{min}$, величина шага $0.02^{\circ}$ в интервале углов $2 \theta$ $5-75^{\circ}$, без вращения образца) в обычной атмосфере.

Диэлектрические свойства композиционных материалов изучали методом импедансной спектроскопии двухконтактным методом. Для осуществления измерений образцы спрессовали в таблетки при давлении $100-120 \mathrm{~kg} / \mathrm{cm}^{2}$. В качестве электродов нанесли $\mathrm{Ag}$ и подклеили медные проволоки. Измерения проводились на воздухе в диапазоне температур $25-160^{\circ} \mathrm{C}$ и частотном диапазоне $10^{-1}-1.4 \cdot 10^{7} \mathrm{~Hz}$ (оптический столик Linkam ETS420 с анализатором импеданса Alpha-AN (Novocontrol, Germany). Обработка полученных данных (подбор эквивалентной схемы) выполнялась при помощи программы Zview.

Изучение объемного распределения фаз в композитных материалах, определение степени однородности и оценку крупности частиц проводили с использованием растрового сканирующего электронного микроскопа Quanta 200 3D (FEI, CША).

Монокристаллы соединения $\mathrm{Cs}_{6} \mathrm{H}\left(\mathrm{HSO}_{4}\right)_{3}\left(\mathrm{H}_{2} \mathrm{PO}_{4}\right)_{4}$ получали методом изотермического упаривания из раствора с мольным соотношением 


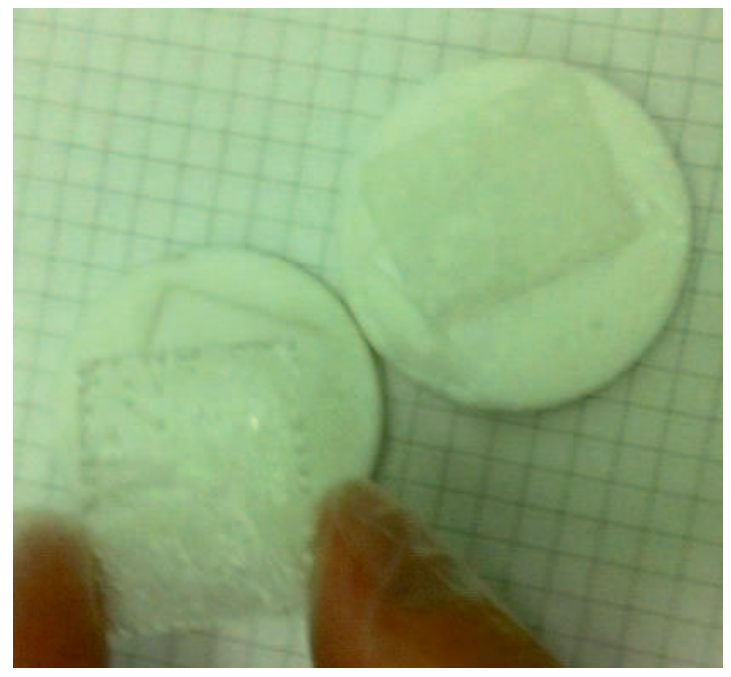

Рис. 1. Образец композиционного материала $(1-x) \mathrm{Cs}_{4}\left(\mathrm{HSO}_{4}\right)_{3}\left(\mathrm{H}_{2} \mathrm{PO}_{4}\right)+x \mathrm{AlPO}_{4} \quad$ после высушивания в форме.

$\mathrm{CsHSO}_{4}: \mathrm{CsH}_{2} \mathrm{PO}_{4}: \mathrm{H}_{3} \mathrm{PO}_{4}$ равным $3: 3: 1$ в условиях, описанных ранее [3]. Фазовый состав полученных монокристаллов контролировали методом РФА.

Получение материала по методике, более подробно описанной в работе [4], и включал в себя стадию гидролиза раствора аммонийалюминиевых квасцов избытком водного раствора аммиака по реакции:

$$
\begin{aligned}
& \mathrm{NH}_{4} \mathrm{Al}\left(\mathrm{SO}_{4}\right)_{2} \cdot 12 \mathrm{H}_{2} \mathrm{O}+\mathrm{NH}_{3} \cdot \mathrm{H}_{2} \mathrm{O} \\
& \rightarrow \mathrm{Al}(\mathrm{OH})_{3} \downarrow+\mathrm{H}_{2} \mathrm{O}+\left(\mathrm{NH}_{4}\right)_{2} \mathrm{SO}_{4}
\end{aligned}
$$

Сразу же после коагуляции осадка проводилось отделение $\mathrm{Al}(\mathrm{OH})_{3} \cdot n \mathrm{H}_{2} \mathrm{O}$ фильтрованием. После определения количества теряемой свежеосажденным гидроксидом алюминия воды проводилась нейтрализация навески $\mathrm{Al}(\mathrm{OH})_{3} \cdot n \mathrm{H}_{2} \mathrm{O}$ фосфорной кислотой по реакции:

$$
\mathrm{Al}(\mathrm{OH})_{3} \cdot n \mathrm{H}_{2} \mathrm{O}+\mathrm{H}_{3} \mathrm{PO}_{4} \rightarrow \mathrm{AlPO}_{4}+2 \mathrm{H}_{2} \mathrm{O}+n \mathrm{H}_{2} \mathrm{O}
$$

После нейтрализации прозрачные растворы фосфата алюминия упаривали до густой сиропообразной консистенции. К полученным растворам добавлялись навески размолотого монокристалла $\mathrm{Cs}_{6} \mathrm{H}\left(\mathrm{HSO}_{4}\right)_{3}\left(\mathrm{H}_{2} \mathrm{PO}_{4}\right)_{4}$ и полученные смеси отливались в специальные гибкие силиконовые формы. После застывания и высушивания отливки в виде тонких $(0.5 \div 1.5 \mathrm{~mm})$ пластин извлекались из форм для проведения дальнейших исследований, рис. 1.

\section{3. Результаты}

Результаты рентгенофазового анализа представлены на рис. 2. При исследовании ряда образцов целевого композита методом рентгенофазового анализа показано, что алюмофосфатный компонент в условиях синтеза образуется в виде аморфной матрицы с некоторой долей кристалличности, что обуславливает прозрачность получаемых отливок образцов.

На рентгенограммах это проявляется в виде диффузионного гало, причем с увеличением доли алюмофосфатного компонента в материале $x \mathrm{Cs}_{6} \mathrm{H}\left(\mathrm{HSO}_{4}\right)_{3}\left(\mathrm{H}_{2} \mathrm{PO}_{4}\right)_{4}$ $+(1-x) \mathrm{AlPO}_{4}$ величина гало возрастает.

Влияние способа получения и состава композитного материала на температурную зависимость проводимости на примере состава $0.9 \mathrm{Cs} 6 \mathrm{H}\left(\mathrm{HSO}_{4}\right)_{3}\left(\mathrm{H}_{2} \mathrm{PO}_{4}\right)_{4}$ $+0.1 \mathrm{AlPO}_{4}$ представлена на рис. 3 .

Фазовый переход в образце является существенно „размытыми“ по температуре в сравнении с исходным монокристаллом. Примечательно, что проводимость при температуре ниже температуры фазово-

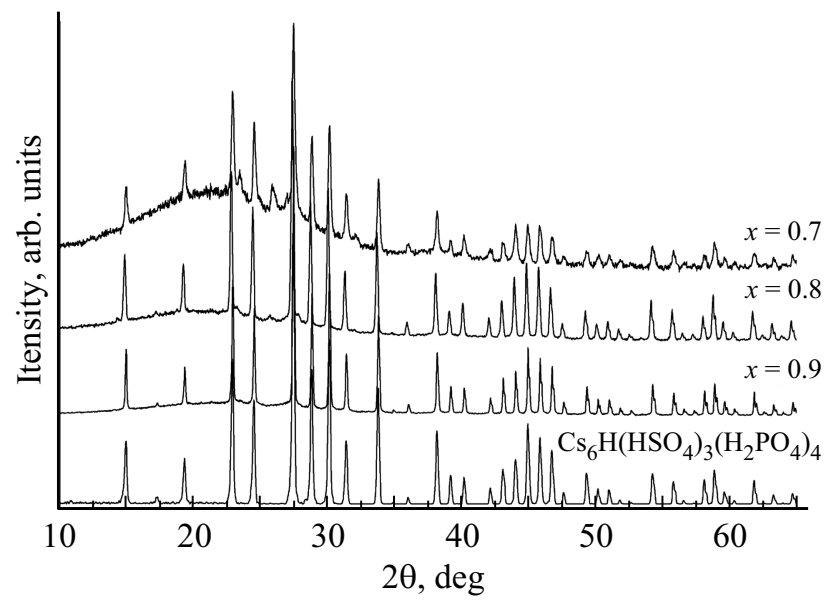

Рис. 2. Сравнение порошковых рентгенограмм фазы $\mathrm{Cs}_{6} \mathrm{H}\left(\mathrm{HSO}_{4}\right)_{3}\left(\mathrm{H}_{2} \mathrm{PO}_{4}\right)_{4}$, материала $x_{\mathrm{Cs}_{6}} \mathrm{H}\left(\mathrm{HSO}_{4}\right)_{3}\left(\mathrm{H}_{2} \mathrm{PO}_{4}\right)_{4}$ $+(1-x) \mathrm{AlPO}_{4}$ с изменением параметра $x$.

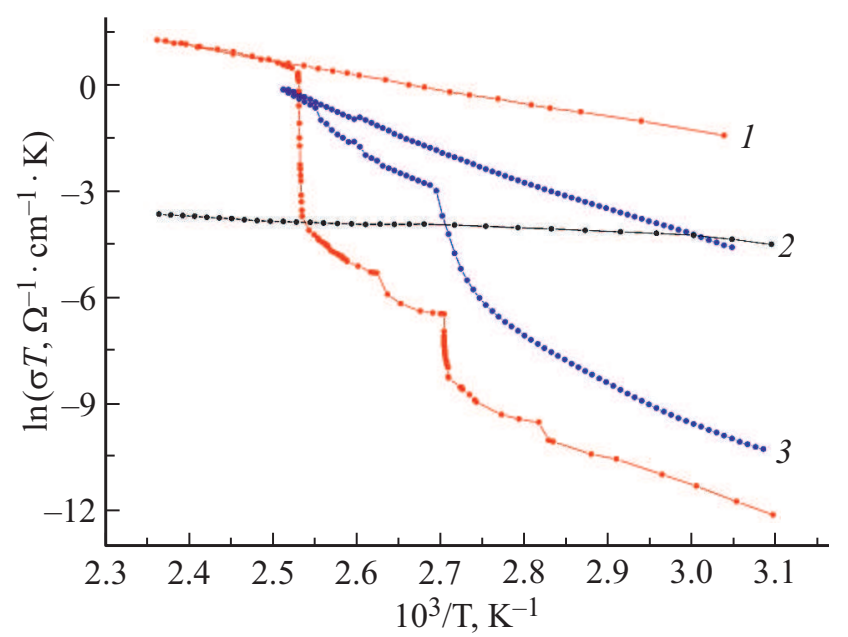

Рис. 3. Сравнение температурной зависимости проводимости исследованных материалов: 1 - исходный монокристалл $\mathrm{Cs}_{6} \mathrm{H}\left(\mathrm{HSO}_{4}\right)_{3}\left(\mathrm{H}_{2} \mathrm{PO}_{4}\right)_{4}, 2$ - свежесинтезированный алюмофосфатный компонент, $3-0.9 \mathrm{Cs}_{6} \mathrm{H}\left(\mathrm{HSO}_{4}\right)_{3}\left(\mathrm{H}_{2} \mathrm{PO}_{4}\right)_{4}-0.1 \mathrm{AlPO}_{4}$ 


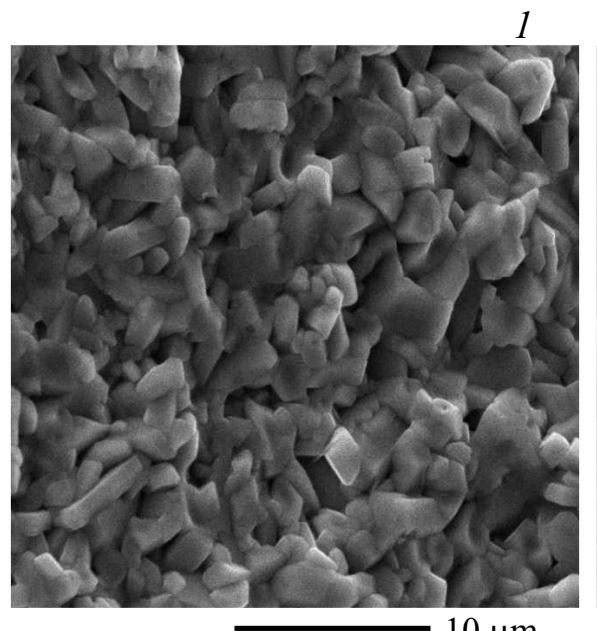

$10 \mu \mathrm{m}$

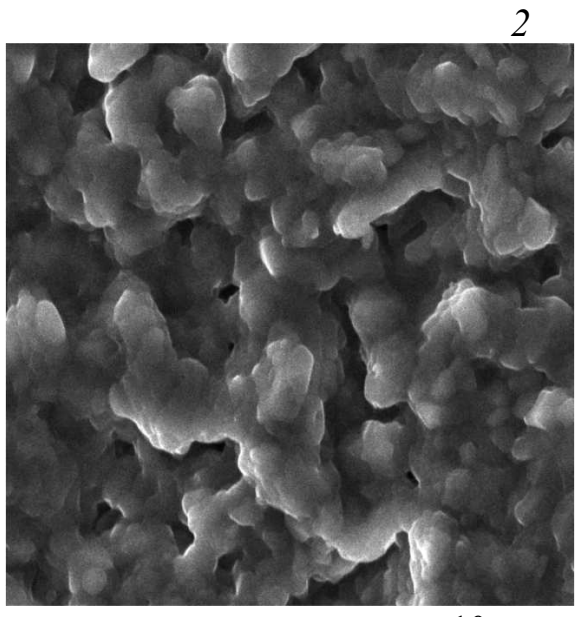

$10 \mu \mathrm{m}$

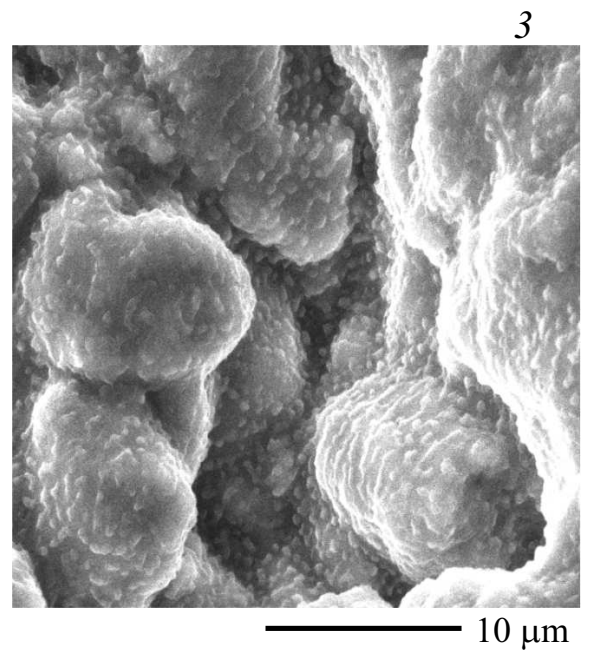

Pис. 4. Электронная микроскопия образцов материала: $1-0.9 \mathrm{Cs}_{6} \mathrm{H}_{(}\left(\mathrm{HSO}_{4}\right)_{3}\left(\mathrm{H}_{2} \mathrm{PO}_{4}\right)_{4}-0.1 \mathrm{AlPO}_{4} ; \quad 2 \quad-$ $0.8 \mathrm{Cs}_{6} \mathrm{H}\left(\mathrm{HSO}_{4}\right)_{3}\left(\mathrm{H}_{2} \mathrm{PO}_{4}\right)_{4}-0.2 \mathrm{AlPO}_{4} ; 3--0.7 \mathrm{Cs}_{6} \mathrm{H}_{(}\left(\mathrm{HSO}_{4}\right)_{3}\left(\mathrm{H}_{2} \mathrm{PO}_{4}\right)_{4}-0.3 \mathrm{AlPO}_{4}$ (нормировочный отрезок $\left.-10 \mu \mathrm{m}\right)$.

го перехода превышает проводимость монокристалла $\mathrm{Cs} 6 \mathrm{H}\left(\mathrm{HSO}_{4}\right)_{3}\left(\mathrm{H}_{2} \mathrm{PO}_{4}\right)_{4}$. При этом проводимость материала $0.9 \mathrm{Cs} 6 \mathrm{H}\left(\mathrm{HSO}_{4}\right)_{3}\left(\mathrm{H}_{2} \mathrm{PO}_{4}\right)_{4}+0.1 \mathrm{AlPO}_{4}$ сопоставима со значением проводимости для монокристалла $\mathrm{Cs}_{6} \mathrm{H}\left(\mathrm{HSO}_{4}\right)_{3}\left(\mathrm{H}_{2} \mathrm{PO}_{4}\right)_{4}$ при температурах выше температуры фазового перехода.

Была измерена и проводимость чистого алюмофосфатного компонента. Фазового перехода при температурах эксперимента данная добавка не проявляет, однако порядок проводимости $\left(10^{-4} \div 10^{-3} \Omega^{-1} \cdot \mathrm{cm}^{-1}\right)$ однозначно указывает на способность алюмофосфатного компонента участвовать в процессе протонного транспорта.

Результаты электронной микроскопии поверхностей образцов отливок, осушенных на воздухе, представлены на рис. 4.

Полученные результаты обусловлены применяемой методикой синтеза образцов. В материалах с $x>0.7$ образуются соприкасающиеся между собой кристаллиты проводящей фазы $\mathrm{Cs}_{6} \mathrm{H}\left(\mathrm{HSO}_{4}\right)_{3}\left(\mathrm{H}_{2} \mathrm{PO}_{4}\right)_{4}$ размерами $0.5 \div 1.5 \mu \mathrm{m}$, пустоты между которыми заполнены армирующей фазой. При дальнейшем увеличении содержания армирующей алюмофосфатной добавки меняются объемные доли фаз, что приводит к уменьшению среднего размера кристаллитов проводящей фазы, и уже при достижении значения $x=0.7$ и менее кристаллиты фазы $\mathrm{Cs}_{6} \mathrm{H}\left(\mathrm{HSO}_{4}\right)_{3}\left(\mathrm{H}_{2} \mathrm{PO}_{4}\right)_{4}$ образуются изолированными друг от друга. Это легко заметить при сравнении снимков разных по составу материалов в одинаковом масштабе.

Полученные результаты хорошо согласуются и объясняют результаты импедансной спектроскопии. При такой пространственной структуре материала (одна фаза заполняет пустоты между частицами другой фазы) межфазная граница является сплошной по объему и способна вносить существенный вклад в проводимость материала.

\section{4. Обсуждение результатов и выводы}

Полученные в процессе исследования экспериментальные результаты однозначно указывают на наличие суперпротонных фазовых переходов в материале $x \mathrm{Cs}_{6} \mathrm{H}\left(\mathrm{HSO}_{4}\right)_{3}\left(\mathrm{H}_{2} \mathrm{PO}_{4}\right)_{4}+(1-x) \mathrm{AlPO}_{4}$. Основные особенности рассматриваемого материала связаны с двумя ключевыми параметрами: способ получения материала $x \mathrm{Cs} 6 \mathrm{H}\left(\mathrm{HSO}_{4}\right)_{3}\left(\mathrm{H}_{2} \mathrm{PO}_{4}\right)_{4}+(1-x) \mathrm{AlPO}_{4}$ (отливка густого раствора в форму с образованием фаз в одном объеме), и свойствами используемого армирующего компонента $\left(\mathrm{AlPO}_{4}\right)$.

Благодаря опробованному подходу к синтезу материала $x \mathrm{Cs}_{6} \mathrm{H}\left(\mathrm{HSO}_{4}\right)_{3}\left(\mathrm{H}_{2} \mathrm{PO}_{4}\right)_{4}+(1-x) \mathrm{AlPO}_{4}$, образование протонпроводящей фазы $\mathrm{Cs}_{6} \mathrm{H}\left(\mathrm{HSO}_{4}\right)_{3}\left(\mathrm{H}_{2} \mathrm{PO}_{4}\right)_{4}$ и фазы $\mathrm{AlPO}_{4}$ происходит в одном объеме.

Применяемый в работе подход к синтезу материала $x \mathrm{Cs}_{6} \mathrm{H}\left(\mathrm{HSO}_{4}\right)_{3}\left(\mathrm{H}_{2} \mathrm{PO}_{4}\right)_{4}+(1-x) \mathrm{AlPO}_{4}$ положительно сказывается на однородности материала и снижении его газопроницаемости по причине отсутствия пор в образцах.

Повышенная при низких температурах проводимость $x \mathrm{Cs}_{6} \mathrm{H}\left(\mathrm{HSO}_{4}\right)_{3}\left(\mathrm{H}_{2} \mathrm{PO}_{4}\right)_{4}+(1-x) \mathrm{AlPO}_{4}$ в сравнении с монокристаллом $\mathrm{Cs}_{6} \mathrm{H}\left(\mathrm{HSO}_{4}\right)_{3}\left(\mathrm{H}_{2} \mathrm{PO}_{4}\right)_{4}$ указывает на дополнительный вклад в проводимость алюмофосфатной добавки наряду с проводимостью по межфазной границе. Это позволяет предполагать способность алюмофосфатной добавки участвовать в процессе протонного транспорта наряду с протонпроводящей фазой $\mathrm{Cs}_{6} \mathrm{H}\left(\mathrm{HSO}_{4}\right)_{3}\left(\mathrm{H}_{2} \mathrm{PO}_{4}\right)_{4}$.

В значительной степени аморфное состояние фазы $\mathrm{AlPO}_{4}$ в составе композиционного материала положительно сказывается на его механических (высокая однородность, прозрачность материала) и проводящих (за счет большей площади межфазной границы, более силь- 
ного поверхностного взаимодействия между частицами разных фаз) свойствах.

\section{Финансирование работы}

Работа выполнена при поддержке Министерства науки и высшего образования РФ в рамках Государственного задания ФНИЦ „Кристаллография и фотоника" РАН в части синтеза композитных материалов $x \mathrm{Cs}_{6} \mathrm{H}\left(\mathrm{HSO}_{4}\right)_{3}\left(\mathrm{H}_{2} \mathrm{PO}_{4}\right)_{4}+(1-x) \mathrm{AlPO}_{4}$. Характеризация физико-химических свойств кристаллов выполнена при финансовой поддержке Российского фонда фундаментальных исследований (проект № 18-32-20050). Работа выполнена с использованием оборудования ЦКП ФНИЦ „Кристаллография и фотоника“ РАН при поддержке Минобрнауки.

\section{Конфликт интересов}

Авторы подтверждают, что у них нет конфликта интересов.

\section{Список литературы}

[1] T. Norby, S.M. Haile, D.A. Boysen, C.R.I. Chisholm, R.V. Merle. Nature 410, 877 (2001).

[2] R. Fitzergerald. Phys. Today. 54, 21 (2001).

[3] В.А. Коморников, В.В. Гребенев, И.П. Макарова, Е.В. Селезнева, П.В. Андреев. Кристаллография 61, 645 (2016).

[4] В.А. Коморников, А.М. Гречихина, В.В. Гребенев, И.С. Тимаков, О.Б. Зайнуллин, В.Г. Зиновьев. Кристаллография 63, 806 (2018).

Редактор Т.Н. Василевская 